\author{
单个、自生的 RP-Au-PR 结构增强金属纳米团簇的 \\ 光致发光量子产率 19 倍 \\ 许道兰 $\uparrow, a, b$ 杨颖 $\uparrow, a, b$ 范文涛 $\uparrow, b, c, a$ 何宗兵 $a, b$ 邹家丰 $a, b$ \\ 冯䂞 $a, b$ 李漫波*,a,b伍志鲲*,a,b \\ ( $a$ 安徽大学 物质科学与信息技术研究院 合肥 230601) \\ ( $b$ 中国科学院固体物理研究所 中国科学院材料物理重点实验室 安徽省纳米材料与技术重点实验室 \\ 中国科学院纳米卓越中心 中国科学院合肥物质科学研究院 合肥 230031) \\ ( $c$ 中国科学技术大学 合肥 230026)
}

\begin{abstract}
摘要 膦保护金纳米团簇的研究可追溯到 1969 年, “RP-Au-PR” ( $R$ 为烷基)结构在膦保护的金纳米团簇上目前还未见报 道, 而相似的 “RS-Au-SR” 结构在硫醇保护金纳米团簇中经常被发现, 其具有多种功能(例如增强团簇发光). 本工作 以双膦作为配体成功合成出原子层次单分布的金银纳米团簇 $\left[\mathrm{Au}_{10} \mathrm{Ag}_{4}(\mathrm{Dppp})_{5} \mathrm{Cl}_{4}\right] \mathrm{Cl}_{2}\left(\mathrm{Au}_{10} \mathrm{Ag}_{4} \mathrm{NC}, \mathrm{Dppp}\right.$ 为 1,3 -双 (二苯 基膦)丙烷), 首次发现它具有 “RP-Au-PR” 结构. 有趣的是, 当二氯甲烷作为溶剂时, 该结构可在 $\mathrm{Au}_{10} \mathrm{Ag}_{4} \mathrm{NC}$ 中自行 消失, 生成 $\left[\mathrm{Au}_{9} \mathrm{Ag}_{4}(\mathrm{Dppp})_{4} \mathrm{Cl}_{4}\right] \mathrm{Cl}\left(\mathrm{Au}_{9} \mathrm{Ag}_{4} \mathrm{NC}\right)$; 另一方面, 当乙醇为溶剂时, 该结构可在 $\mathrm{Au}_{9} \mathrm{Ag}_{4} \mathrm{NC}$ 中自行生成, 从而 转变成 $\mathrm{Au}_{10} \mathrm{Ag}_{4} \mathrm{NC}$. 两个团簇的紫外-可见-近红外(UV/Vis/NIR)吸收峰除了有轻微的位移外, 没有显著的区别, 暗示着 “RP-Au-PR” 结构对团簇的电子结构没有根本影响, 这一点也被密度泛函理论(DFT)计算所支撑. 该结构促使 $\mathrm{Au}_{10} \mathrm{Ag} 4$ $\mathrm{NC}$ 的光致发光量子产率比 $\mathrm{Au}_{9} \mathrm{Ag}_{4} \mathrm{NC}$ 的提高了 19 倍(达到 10.32\%), 这归因于 “RP-Au-PR” 结构诱导的电荷转移和刚 性的增强. 本工作揭示了新的团簇结构和发光现象, 对团簇构效关系的理解和性能改善有重要的启发.
\end{abstract}

关键词 金属纳米团簇; “RP-Au-PR” 结构; 光致发光

\title{
Single, Self-Born RP-Au-PR Motif Boosts 19-Fold Photoluminescence Quantum Yield of Metal Nanocluster
}

\author{
$\mathrm{Xu}$, Daolan ${ }^{\dagger, a, b}$ \\ Yang, Ying ${ }^{\dagger a, b}$ \\ Feng, Lei ${ }^{a, b}$ \\ Fan, Wentao $0^{\dagger, b, c, a}$ \\ $\mathrm{Li}$, Man-Bo*,a,b \\ He, Zongbing ${ }^{a, b}$
Wu, Zhikun*,a,b \\ Zou, Jiafeng ${ }^{a, b}$ \\ ( ${ }^{a}$ Institute of Physical Science and Information Technology, Anhui University, Hefei 230601, China) \\ $\left({ }^{b}\right.$ Key Laboratory of Materials Physics, Anhui Key Laboratory of Nanomaterials and Nanotechnology, CAS Center for \\ Excellence in Nanoscience, Institute of Solid State Physics, Hefei Institutes of Physical Science, \\ Chinese Academy of Sciences, Hefei 230031, China) \\ ( ${ }^{c}$ University of Science and Technology of China, Hefei 230026, China)
}

\begin{abstract}
The study of phosphine-protected gold nanocluster can be dated back to 1969, however, the RP-Au-PR motif has not been reported until now. Meanwhile, its analogue RS-Au-SR motif was often reported in thiolated gold nanoclusters, being of multiple functions (e.g., enhancing the luminescence). Herein, we successfully synthesized a monodisperse gold-silver nanocluster: $\left[\mathrm{Au}_{10} \mathrm{Ag}_{4}(\mathrm{Dppp})_{5} \mathrm{Cl}_{4}\right] \mathrm{Cl}_{2}\left(\mathrm{Au}_{10} \mathrm{Ag}_{4} \mathrm{NC}\right.$, where the Dppp represents 1,3-diphenylphosphine propane, and $\mathrm{NC}$ represents nanocluster) by introducing the reducing agent $\left(\mathrm{NaBH}_{4}\right)$ in an dichloromethane-ethanol solution of chloroauric acid, silver nitrate and 1,3-diphenylphosphine propane. Hydrochloric acid was added to promote the nanocluster size-focusing. $\mathrm{Au}_{10} \mathrm{Ag}_{4} \mathrm{NC}$ is unstable in dichloromethane and will be transformed into another nanocluster $\left[\mathrm{Au}_{9} \mathrm{Ag}_{4}(\mathrm{Dppp})_{4} \mathrm{Cl}_{4}\right] \mathrm{Cl}\left(\mathrm{Au}_{9} \mathrm{Ag}_{4} \mathrm{NC}\right)$. The structures and compositions of $\mathrm{Au}_{10} \mathrm{Ag}_{4}$ and $\mathrm{Au}_{9} \mathrm{Ag}_{4} \mathrm{NCs}_{\text {have }}$ been determined by single crystal X-ray crystallography and electrospray ionization mass spectrometry (ESI-MS). $\mathrm{Au}_{10} \mathrm{Ag}_{4} \mathrm{NC}$ possesses an ico-
\end{abstract}

\footnotetext{
*E-mail: mbli@ahu.edu.cn; zkwu@issp.ac.cn

$\dagger$ These authors contributed equally to this work.

Received November 5, 2021; published November 17, 2021.

Supporting information for this article is available free of charge via the Internet at http://sioc-journal.cn.

Project supported by the National Natural Science Foundation of China (Nos. 92061110, 21925303, 21771186, 21829501, 21222301, 21171170, and 21528303), the Hefei National Laboratory for Physical Sciences at the Microscale (No. KF2020102), the Anhui University (No. S020118002/113 and the provision of Confotec ${ }^{\mathrm{TM}}$ MR520 fluorescence/Raman spectrometer), the CASHIPS Director's Fund (No. BJPY2019A02), the Key Program of the 13th five-year plan, CASHIPS (No. KP-2017-16), and the Collaborative Innovation Program of Hefei Science Center, CAS (No. 2020HSC-CIP005).

项目受国家自然科学基金(Nos. 92061110, 21925303, 21771186, 21829501, 21222301, 21171170 和 21528303)、合肥微尺度物质科学国家研究中心(No. KF2020102)、安徽大学(No. S020118002/113 以及配备 Confotec ${ }^{\mathrm{TM}} \mathrm{MR} 520$ 苂光/拉曼光谱仪)、中国科学院合肥研究院院长基金(No. BJPY2019A02)、 中科院合肥物质科学研究院 “十三五” 规划重点支持项目(No. KP-2017-16)和中国科学院合肥科学中心协同创新项目(No. 2020HSC-CIP005)资助.
} 
sahedral $\mathrm{Au}_{9} \mathrm{Ag}_{4}$ core, which is protected by a special RP-Au-PR motif, diphosphine and halogen ligands; $\mathrm{Au}_{9} \mathrm{Ag}_{4} \mathrm{NC}_{\mathrm{C}}$ as an icosahedral $\mathrm{Au}_{9} \mathrm{Ag}_{4}$ metal core protected by bisphosphine and halogen ligands, thus the major structural difference between $\mathrm{Au}_{10} \mathrm{Ag}_{4}$ and $\mathrm{Au}_{9} \mathrm{Ag}_{4} \mathrm{NCs}$ is the RP-Au-PR motif. The motif can be self-vanished in $\mathrm{Au}_{10} \mathrm{Ag}_{4} \mathrm{NC}$ when dichloromethane acts as the solvent, resulting in the formation of $\mathrm{Au}_{9} \mathrm{Ag}_{4} \mathrm{NC}$ as mentioned above; on the other hand, the motif can self-bear in $\mathrm{Au}_{9} \mathrm{Ag}_{4} \mathrm{NC}$ when the solvent is ethanol instead of dichloromethane, resulting in the formation of $\mathrm{Au}_{10} \mathrm{Ag}_{4} \mathrm{NC}_{\text {. The two }}$ nanoclusters showed similar ultraviolet-visible-near infrared (UV/Vis/NIR) absorption spectra except for slight shift of the profile, indicating that the RP-Au-PR motif has no essential influence on the electronic structure of nanocluster, which was supported by density functional theory (DFT) calculations. However, the photoluminescence quantum yield of $\mathrm{Au}_{10} \mathrm{Ag}_{4} \mathrm{NC}$ $(10.32 \%)$ is 19 times higher than that of $\mathrm{Au}_{9} \mathrm{Ag}_{4} \mathrm{NC}(0.52 \%)$, ascribing to the enhancement of charge transfer and structure rigidity induced by the motif. This work provides new views to the structure and photoluminescence of gold-silver nanocluster, has important implications for the structure-property understanding and the property improvement.

Keywords metal nanocluster; RP-Au-PR motif; photoluminescence

\section{1 引言}

金属纳米团簇由于具有精确的原子组成与结构、超 小的纳米尺寸 $(3 \mathrm{~nm}$ 以下)和丰富的物理化学性质 $[1-8]$, 在催化 ${ }^{[9-13]}$ 、生物医学 ${ }^{[14-18]}$ 和传感 ${ }^{[19-23]}$ 等领域具有极大 的应用潜力, 引起了科学家们的广泛关注. 稳定性是团 簇科学研究和实际应用中需要考察的一个重要因素, 这 也是科学家们近来将注意力从膦保护的金属纳米团簇 转移到硫醇保护的金属纳米团簇的原因. 实际上, 前者 比后者有着更悠久的研究历史 ${ }^{[24-29]}$. 硫醇配体保护的金 属纳米团簇中经常出现的 “RS-Au-SR” 结构 ( $\mathrm{R}$ 为烷基) 不仅起着稳定团簇的作用 ${ }^{[30-31]}$, 还可以通过 $M-S(M$ 为金属原子)键的电荷转移增强金属纳米团簇的光致发 光 $(\mathrm{PL})^{[32-34]}$. 遗憾的是, 到目前为止, 在有机膦保护的 金属纳米团簇中还没有报道过 “RP-Au-PR” 结构, 可 能由于 P(III) 的半径相对较小、 $\mathrm{P}$ 原子周围的空间位阻 较大等原因导致. 具有较小空间位阻和多个配位点的多 齿膦配体可能有助于形成这种结构, 这一点被我们的实 验所证实. 下面我们将阐述一些细节情况.

\section{2 结果与讨论}

通常的合成操作为: 将 $\mathrm{HAuCl}_{4} \cdot 4 \mathrm{H}_{2} \mathrm{O} 、 \mathrm{AgNO}_{3}$ 和 1,3-双 (二苯基膦) 丙烷 (Dppp)溶解在二氯甲烷和乙醇的 混合溶剂中, 经硼氢化钠还原后, 加入浓盐酸进行尺寸

a

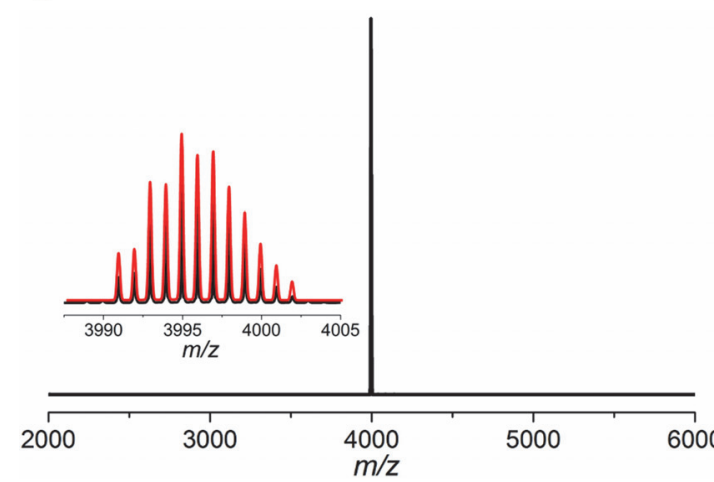

聚焦 ${ }^{[35-36]}$ (支持信息，图 S1). 盐酸的这种功能在双膦配 体保护的金纳米团簇合成中已有体现 ${ }^{[37]}$. 粗产物用大 量的水和乙醚洗涤多次, 除去未反应的酸、盐和双膦配 体, 得到比较纯净的产物. 通过将戊烷扩散到含有产物 的乙醇溶液中, 静置大约两周后, 析出红橙色的块状晶 体。通过 $\mathrm{X}$ 射线衍射分析, 确定它为 $\left[\mathrm{Au}_{10} \mathrm{Ag}_{4}(\mathrm{Dppp})_{5} \mathrm{Cl}_{4}\right] \mathrm{Cl}_{2}$ (简称 $\mathrm{Au}_{10} \mathrm{Ag}_{4} \mathrm{NC}$ ). 在实验过程 中, 发现它在二氯甲烷中不稳定, 会逐渐转变成另一种 纳米团簇 $\left[\mathrm{Au}_{9} \mathrm{Ag}_{4}(\mathrm{Dppp})_{4} \mathrm{Cl} 4 \mathrm{Cl}\right.$ (简称 $\mathrm{Au}_{9} \mathrm{Ag}_{4} \mathrm{NC}$ ). 通过 在二氯甲烷和正己烷的混合溶剂中慢慢扩散, 大约两周 后出现它的黄橙色针状晶体.

$\mathrm{Au}_{9} \mathrm{Ag}_{4} \mathrm{NC}$ 和 $\mathrm{Au}_{10} \mathrm{Ag}_{4} \mathrm{NC}$ 通过电喷雾电离质谱 (ESI-MS) 在正离子模式下进行表征. $\mathrm{Au}_{9} \mathrm{Ag}_{4} \mathrm{NC}$ 在 $\mathrm{m} / \mathrm{z}$ $=3994.97$ 处有一个强的正一价离子峰(图 1a), 归属于 $\left[\mathrm{Au}_{9} \mathrm{Ag}_{4}(\mathrm{Dppp})_{4} \mathrm{Cl}_{4}\right]^{+}$. 对于 $\mathrm{Au}_{10} \mathrm{Ag}_{4} \mathrm{NC}$, 可以观察到在 $m / z=2302.05$ 和 4641.10 处有两个强的信号峰(图 1b), 分 别归属于正二价 $\left[\mathrm{Au}_{10} \mathrm{Ag}_{4}(\mathrm{Dppp})_{5} \mathrm{Cl}_{4}\right]^{2+}$ 和正一价 $\left\{\left[\mathrm{Au}_{10} \mathrm{Ag}_{4}(\mathrm{Dppp})_{5} \mathrm{Cl}_{4}\right] \mathrm{Cl}\right\}^{+}$离子峰. 二者模拟的同位素分 布与实验结果非常吻合(图 1a 和图 $1 \mathrm{~b}$ 的插图), 进一步 表明所归属的分子组成是正确的. 根据超原子理论, $\mathrm{Au}_{9} \mathrm{Ag}_{4}\left[9\left(\mathrm{Au}_{6} \mathrm{~s}^{1}\right)+4\left(\mathrm{Ag} 5 \mathrm{~s}^{1}\right)-4(\mathrm{Cl})-1=8\right]$ 和 $\mathrm{Au}_{10} \mathrm{Ag}_{4}$ $\left[10\left(\mathrm{Au}^{1} \mathrm{~s}^{1}\right)+4\left(\mathrm{Ag} 5 \mathrm{~s}^{1}\right)-4(\mathrm{Cl})-2=8\right]$ 均属于稳定的八电 子体系 ${ }^{[38-42]}$.

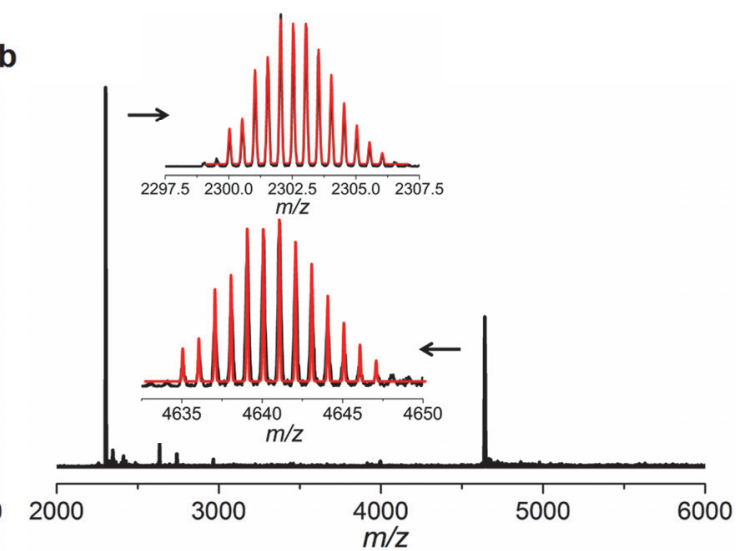

图 $1 \mathrm{Au}_{9} \mathrm{Ag}_{4} \mathrm{NC}$ (a)和 $\mathrm{Au}_{10} \mathrm{Ag}_{4} \mathrm{NC}$ (b)的电喷雾电离质谱, 插图: 实验(黑色)和模拟(红色)同位素分布的对比

Figure 1 ESI-MS of $\mathrm{Au}_{9} \mathrm{Ag}_{4} \mathrm{NC} \mathrm{(a)} \mathrm{and} \mathrm{Au}_{10} \mathrm{Ag}_{4} \mathrm{NC}$ (b), inset: the comparison of the experimental (black) and simulated (red) isotope patterns 
$\mathrm{X}$-射线晶体学揭示了 $\mathrm{Au}_{9} \mathrm{Ag}_{4} \mathrm{NC}$ 和 $\mathrm{Au}_{10} \mathrm{Ag}_{4} \mathrm{NC}$ 的 原子结构. $\mathrm{Au}_{9} \mathrm{Ag}_{4} \mathrm{NC}$ 结晶于单斜晶系 $P 2_{1} / c$ 空间群, 单 个晶胞中含有四个团簇粒子, 而 $\mathrm{Au}_{10} \mathrm{Ag}_{4} \mathrm{NC}$ 结晶于三 斜晶系 $P-1$ 空间群, 单个晶胞中含有两个团簇粒子(支持 信息, 图 S2). 如图 2 和图 S3(支持信息)所示, $\mathrm{Au}_{9} \mathrm{Ag}_{4}$ $\mathrm{NC}$ 有一个二十面体 $\mathrm{Au}_{9} \mathrm{Ag}_{4}$ 内核, 外围被四个 Dppp 和 四个 $\mathrm{Cl}$ 配体覆盖. 四个 Dppp 配体与八个金原子配位, 四个 $\mathrm{Cl}$ 配体与四个银原子配位. $\mathrm{Au}_{10} \mathrm{Ag}_{4} \mathrm{NC}$ 具有与 $\mathrm{Au}_{9} \mathrm{Ag}_{4} \mathrm{NC}$ 相似的金属内核, 由一个 Dppp-Au-Dppp 结 构、三个 Dppp 和四个 $\mathrm{Cl}$ 配体保护着. $\mathrm{Au}_{10} \mathrm{Ag}_{4} \mathrm{NC}$ 的总 体结构比 $\mathrm{Au}_{9} \mathrm{Ag}_{4} \mathrm{NC}$ 的多一个 $\mathrm{Au}-\mathrm{Dppp}-\mathrm{Cl}$ 单元. 在这 两个纳米团簇中, 所有的金原子均与 Dppp 配位, 而所 有的银原子均与 $\mathrm{Cl}$ 配位, 这种配位倾向可以通过泡利 电负性大小来解释. 由于金的泡利电负性(2.54)高于银 的泡利电负性(1.93), 故金原子优先占据电子密度高的 区域, 即与给电子的有机膦结合, 而银原子和吸电子的 卤素结合. 这种结构相对于其它结构更稳定, 这可能也 是能获得原子层次单分布金银纳米团簇的原因所在.

值得注意的是, 首次在膦保护的金属纳米团簇中观 察到 Dppp-Au-Dppp 结构. 而且, 该结构是 $\mathrm{Au}_{9} \mathrm{Ag}_{4} \mathrm{NC}$ 和 $\mathrm{Au}_{10} \mathrm{Ag}_{4} \mathrm{NC}$ 之间的主要结构差异. 为了研究该结构 对纳米团簇电子结构的影响, 我们对二者进行了紫外可见-近红外(UV/Vis/NIR)吸收光谱测试, 观察到二者 表现出相似的光谱, 只是光谱的形状和峰位有些微差 别. 具体而言, $\mathrm{Au}_{9} \mathrm{Ag}_{4} \mathrm{NC}$ 的光谱在 321 和 $420 \mathrm{~nm}$ 处有
两个突出的峰，而 $\mathrm{Au}_{10} \mathrm{Ag}_{4} \mathrm{NC}$ 的光谱在 316 和 $415 \mathrm{~nm}$ 处有两个明显的峰, 并且 $\mathrm{Au}_{9} \mathrm{Ag}_{4}$ 和 $\mathrm{Au}_{10} \mathrm{Ag}_{4} \mathrm{NCs}$ 分别在 $365,520 \mathrm{~nm}$ 和 333, $530 \mathrm{~nm}$ 处有两个肩峰(支持信息, 图 S4). 观察图 S4 的插图, 可以确定 $\mathrm{Au}_{9} \mathrm{Ag}_{4}$ 和 $\mathrm{Au}_{10} \mathrm{Ag}_{4}$ $\mathrm{NCs}$ 的光学能隙分别为 2.23 和 $2.14 \mathrm{eV}$.

为深入探究 $\mathrm{Au}_{9} \mathrm{Ag}_{4} \mathrm{NC}$ 和 $\mathrm{Au}_{10} \mathrm{Ag}_{4} \mathrm{NC}$ 的电子结构 差异, 我们对二者的 UV/Vis/NIR 吸收光谱和 Kohn-Sham 轨道进行了密度泛函理论计算。对于 $\mathrm{Au}_{9} \mathrm{Ag}_{4} \mathrm{NC}$ 而言, 在 $420 \mathrm{~nm}$ ( $\beta$, 计算值: $407.27 \mathrm{~nm}$ ) 和 $321 \mathrm{~nm}(\delta$, 计算值: $300.62 \mathrm{~nm}$ )处的主要吸收峰分别对 应于 $\mathrm{HOMO}-1 \rightarrow \mathrm{LUMO}+4$ 和 $\mathrm{HOMO}-2 \rightarrow \mathrm{LUMO}+7$ 电子跃迁; 在 $520 \mathrm{~nm}(\alpha$, 计算值: $462.04 \mathrm{~nm}$ ) 和 $365 \mathrm{~nm}$ ( $\gamma$, 计算值: $344.03 \mathrm{~nm}$ )处的肩峰分别对应于 HOMO$2 \rightarrow$ LUMO $(\mathrm{HOMO} \rightarrow \mathrm{LUMO}+1)$ 和 $\mathrm{HOMO}-5 \rightarrow \mathrm{LUMO}$ 电子跃迁(图 3a). $\mathrm{Au}_{10} \mathrm{Ag}_{4} \mathrm{NC}$ 在 $415 \mathrm{~nm}$ ( $\beta$, 计算值: $406.57 \mathrm{~nm})$ 和 $316 \mathrm{~nm}$ ( $\delta$, 计算值: $299.36 \mathrm{~nm}$ ) 处的主要吸 收峰分别来自 $\mathrm{HOMO}-1 \rightarrow \mathrm{LUMO}+3$ (HOMO $2 \rightarrow \mathrm{LUMO}+1)$ 和 $\mathrm{HOMO}-9 \rightarrow \mathrm{LUMO}+4$ 电子跃迁; 在 $530 \mathrm{~nm}$ ( $\alpha$, 计算: $471.21 \mathrm{~nm}$ )和 $333 \mathrm{~nm}$ ( $\gamma$, 计算: 314.26 $\mathrm{nm})$ 处的肩峰分别来自 $\mathrm{HOMO}-1 \rightarrow \mathrm{LUMO}+1$ $(\mathrm{HOMO} \rightarrow \mathrm{LUMO}+2)$ 和 $\mathrm{HOMO}-7 \rightarrow \mathrm{LUMO}+3$ 电子跃 迁(图 3b). 从它们的 Kohn-Sham 轨道、 HOMOs 和 LUMOs 的电子密度分布(图 3c、图 3d 和图 S5), 可以得 出 Dppp-Au-Dppp 结构对纳米团簇的电子结构没有本质 上的影响.

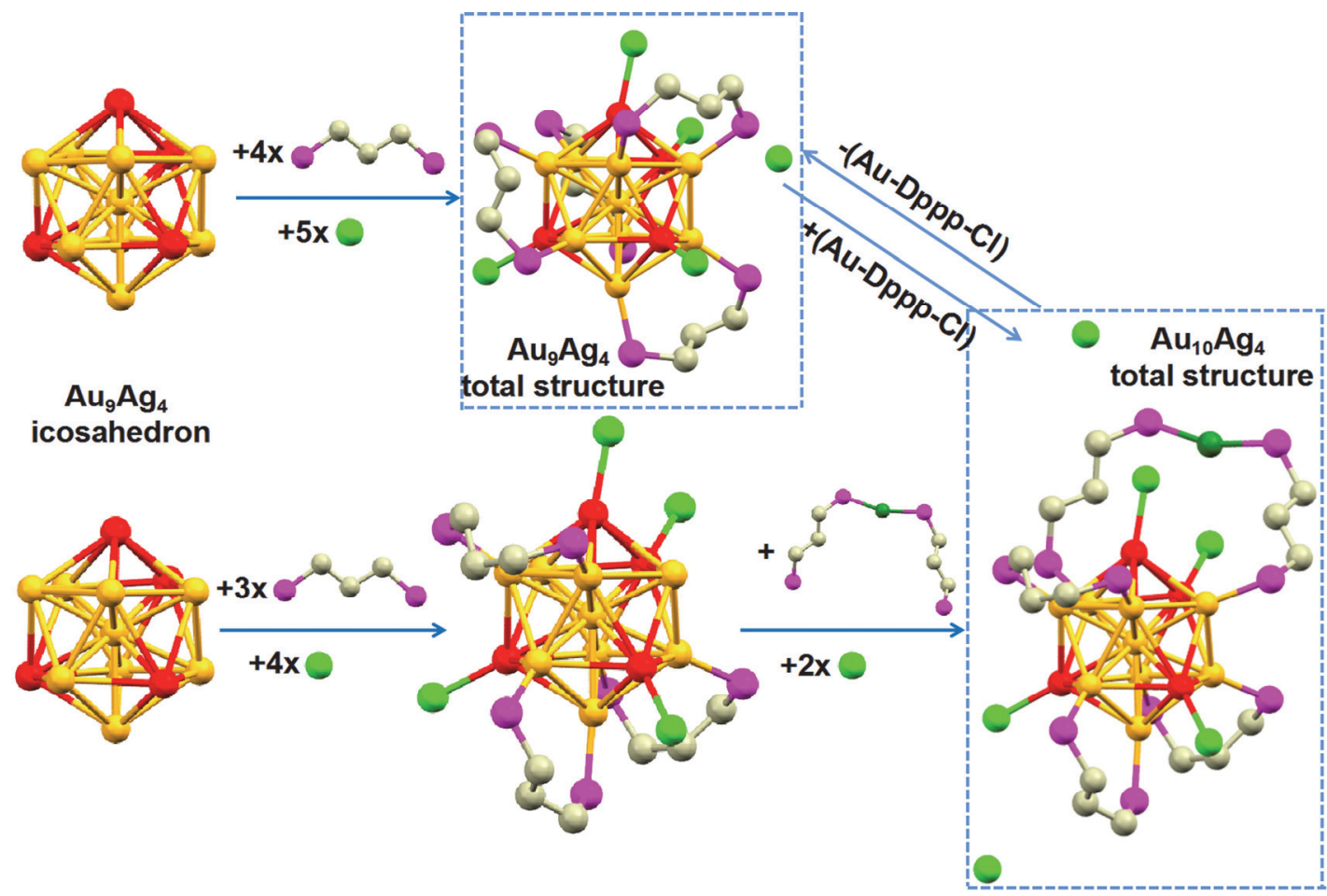

图 $2 \mathrm{Au}_{9} \mathrm{Ag}_{4} \mathrm{NC}$ 和 $\mathrm{Au}_{10} \mathrm{Ag}_{4} \mathrm{NC}$ 的结构示意图. 颜色代码: 橙色、深绿色, $\mathrm{Au}$; 红色, $\mathrm{Ag}$; 紫色, $\mathrm{P} ;$ 亮绿色, $\mathrm{Cl}$; 浅灰色, $\mathrm{C}$. 为了清晰起见, 省略了 所有的苯环和 $\mathrm{H}$ 原子

Figure 2 Schematic illustration of $\mathrm{Au}_{9} \mathrm{Ag}_{4} \mathrm{NC}$ and $\mathrm{Au}_{10} \mathrm{Ag}_{4} \mathrm{NC}$ structures. Color legend: orange, dark green, $\mathrm{Au}$; red, $\mathrm{Ag}$; purple, $\mathrm{P}$; bright green, $\mathrm{Cl}$; gray, $\mathrm{C}$. All benzene rings and $\mathrm{H}$ atoms are omitted for clarity 


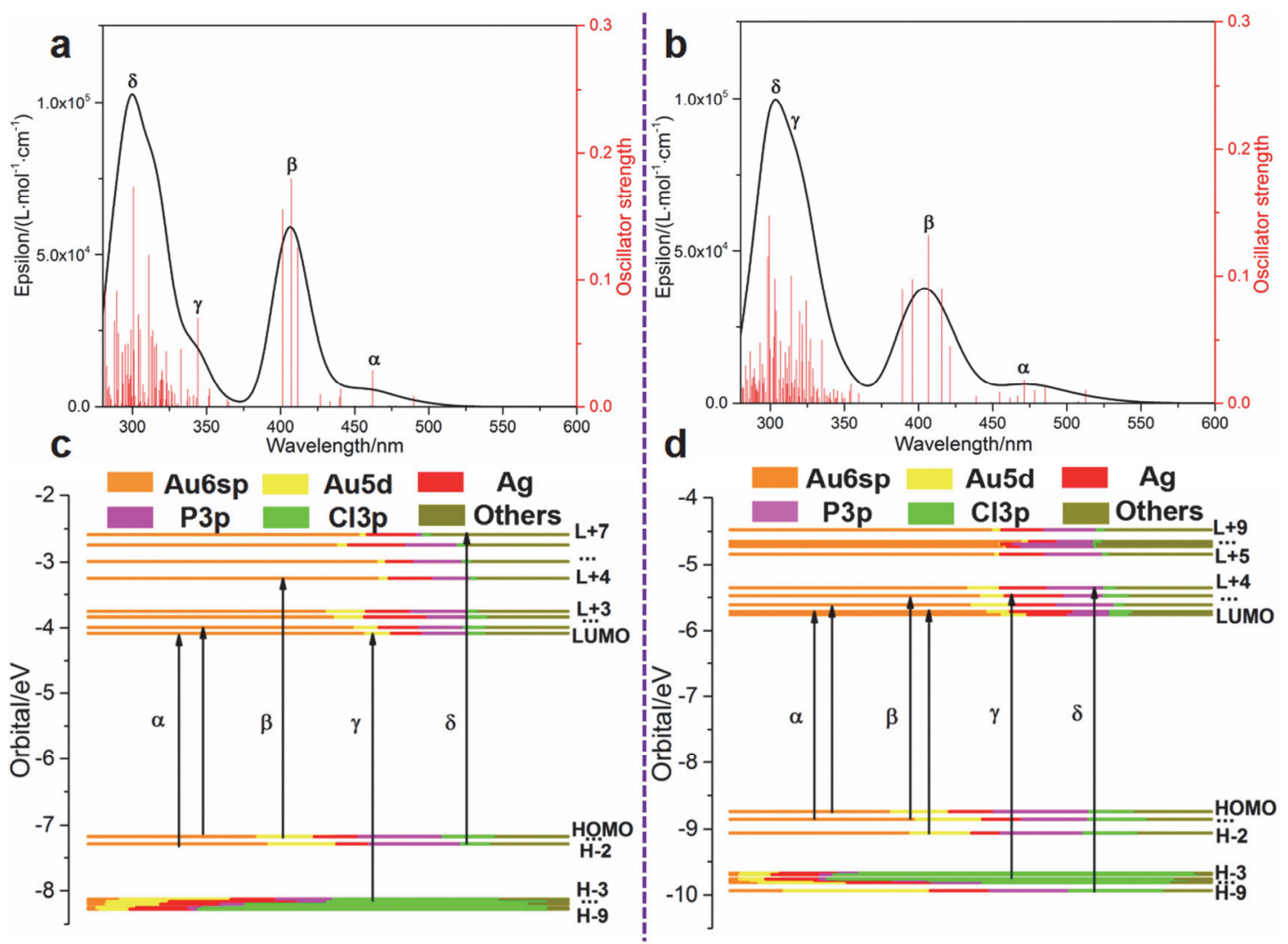

图 3 计算的 $\mathrm{Au}_{9} \mathrm{Ag}_{4} \mathrm{NC}$ (a) 和 $\mathrm{Au}_{10} \mathrm{Ag}_{4} \mathrm{NC}$ (b) 的 UV/Vis/NIR 吸收光谱. $\mathrm{Au}_{9} \mathrm{Ag}_{4} \mathrm{NC}$ (c) 和 $\mathrm{Au}_{10} \mathrm{Ag}_{4} \mathrm{NC}$ (d) 的 Kohn-Sham 轨道

Figure 3 Calculated UV/Vis/NIR absorption spectra of $\mathrm{Au}_{9} \mathrm{Ag}_{4} \mathrm{NC}$ (a) and $\mathrm{Au}_{10} \mathrm{Ag}_{4} \mathrm{NC}$ (b). Kohn-Sham orbitals of $\mathrm{Au}_{9} \mathrm{Ag}_{4} \mathrm{NC}$ (c) and $\mathrm{Au}_{10} \mathrm{Ag}_{4} \mathrm{NC}$ (d)

然而, Dppp-Au-Dppp 结构对于 $\mathrm{Au}_{9} \mathrm{Ag}_{4} \mathrm{NC}$ 和 $\mathrm{Au}_{10} \mathrm{Ag}_{4} \mathrm{NC}$ 的光致发光(PL)量子产率有很大的影响: 在 乙腈中, $\mathrm{Au}_{10} \mathrm{Ag}_{4} \mathrm{NC}$ 的绝对量子产率(10.32\%)比 $\mathrm{Au}_{9} \mathrm{Ag}_{4}$ $\mathrm{NC}$ 的( $0.52 \%)$ 提高了约 19 倍. $\mathrm{Au}_{9} \mathrm{Ag}_{4} \mathrm{NC}$ 和 $\mathrm{Au}_{10} \mathrm{Ag}_{4} \mathrm{NC}$ 的激发光谱与它们各自的 UV/Vis/NIR 吸收光谱非常相 似(图 4a). $\mathrm{Au}_{9} \mathrm{Ag}_{4} \mathrm{NC}$ 和 $\mathrm{Au}_{10} \mathrm{Ag}_{4} \mathrm{NC}$ 的发射峰分别位于 755 和 $735 \mathrm{~nm}$ (图 4b). 这些事实表明, 发光强度的差异 可能部分来源于发射动力学. 进一步的实验支持了这一 观点: $\mathrm{Au}_{9} \mathrm{Ag}_{4} \mathrm{NC}$ 和 $\mathrm{Au}_{10} \mathrm{Ag}_{4} \mathrm{NC}$ 的发光寿命均达到微秒 级别, 但 $\mathrm{Au}_{10} \mathrm{Ag}_{4} \mathrm{NC}$ 的平均发光寿命 $(6.69 \mu \mathrm{s})$ 是 $\mathrm{Au}_{9} \mathrm{Ag}_{4} \mathrm{NC}(2.10 \mu \mathrm{s})$ 的三倍. $\mathrm{Au}_{9} \mathrm{Ag}_{4} \mathrm{NC}$ 拥有三个 PL 寿 命 $t_{1}(0.30 \mu \mathrm{s}), t_{2}(1.87 \mu \mathrm{s})$ 和 $t_{3}(6.97 \mu \mathrm{s})$, 分别占比 $67.10 \%, 7.75 \%$ 和 $25.15 \%$ (图 4c). 然而, $\mathrm{Au}_{10} \mathrm{Ag}_{4} \mathrm{NC}$ 拥有 两个 PL 寿命 $t_{1}(0.36 \mu \mathrm{s})$ 和 $t_{2}(6.91 \mu \mathrm{s})$, 分别占比 $3.33 \%$ 和 $96.67 \%$ (图 4d). 这种情况可能由于 Dppp-Au-Dppp 结 构影响了配体到金属核的电荷转移过程, 从而改变了整 个发射动力学.

$\mathrm{Au}_{10} \mathrm{Ag}_{4} \mathrm{NC}$ 具有更强 PL 的另一个原因可能来自 Dppp-Au-Dppp 结构导致纳米团簇整体结构刚性的增强, 减少表面和内核上各原子的运动, 从而提高纳米团簇的 PL 量子产率: (1) 在 $\mathrm{Au}_{10} \mathrm{Ag}_{4} \mathrm{NC}$ 中, Dppp-Au-Dppp 结构 上的所有金原子和磷原子几乎在同一平面内(支持信息, 图 S6a), 共平面性增加了该团簇的刚性. 这种情况在有
机分子中也能观察到，例如，荧光素 $(\Phi=0.92)$ /酚酞 $(\Phi$ $=0)$ 和芴 $(\Phi=1) /$ 联苯 $(\Phi=0.18$, 支持信息, 图 S7) 呈现 了极大的发光量子产率差异; (2) 该结构的周围存在几 类弱相互作用，包括 $\mathrm{Au} \cdots \mathrm{Cl} 、 \mathrm{Au} \cdots \mathrm{H}-\mathrm{C}$ (苯环氢原子) 和 $\pi \cdots \pi$ 相互作用(支持信息, 图 S6b). 电荷转移和刚性 的增强可以反映在微小的结构变化上. 通过仔细观察, 我们发现 $\mathrm{Au}_{10} \mathrm{Ag}_{4}$ 和 $\mathrm{Au}_{9} \mathrm{Ag}_{4} \mathrm{NCs}$ 的二十面体金属核上 的平均键长不同: $\mathrm{Au}$ 表面一 $\mathrm{Au}$ 表面 $\left(\mathrm{Au}_{9} \mathrm{Ag}_{4}\right.$ 为 $0.289 \mathrm{~nm}$, $\mathrm{Au}_{10} \mathrm{Ag}_{4}$ 为 $\left.0.287 \mathrm{~nm}\right)$ 和 $\mathrm{Au}$ 表面一 $\mathrm{Ag}$ 表面 $\left(\mathrm{Au}_{9} \mathrm{Ag}_{4}\right.$ 为 0.291 $\mathrm{nm}, \mathrm{Au}_{10} \mathrm{Ag}_{4}$ 为 $0.293 \mathrm{~nm}$ ) 有一定差别, 而 $\mathrm{Ag}$ 衣面一 $\mathrm{Ag}$ 衰面 的键长差别更大 $\left(\mathrm{Au}_{9} \mathrm{Ag}_{4}\right.$ 为 $0.299 \mathrm{~nm}, \mathrm{Au}_{10} \mathrm{Ag}_{4}$ 为 0.283 $\mathrm{nm}$ ，见支持信息，图 S8).

当 $\mathrm{Au}_{10} \mathrm{Ag}_{4} \mathrm{NC}$ 溶于二氯甲烷时, Dppp-Au-Dppp 结 构可以自行消失(图 5a), 导致约 95\%的 PL 损失, 转变成 $\mathrm{Au}_{9} \mathrm{Ag}_{4} \mathrm{NC}$; 另一方面，当 $\mathrm{Au}_{9} \mathrm{Ag}_{4} \mathrm{NC}$ 溶于乙醇且升高 温度时, 该结构可以自行生成, 转变成 $\mathrm{Au}_{10} \mathrm{Ag}_{4} \mathrm{NC}$ (图 $5 b$ ，实验细节见支持信息)，导致 PL 增强 19 倍(见图 4b). 换言之, 这两个纳米团簇之间可以相互转变, 并伴随着 PL 增强或减弱, 这种特性可能在传感和转换器领域中 有潜在的应用.

\section{3 结论}

总之, 我们合成了两个新颖的金银纳米团簇, 解出 

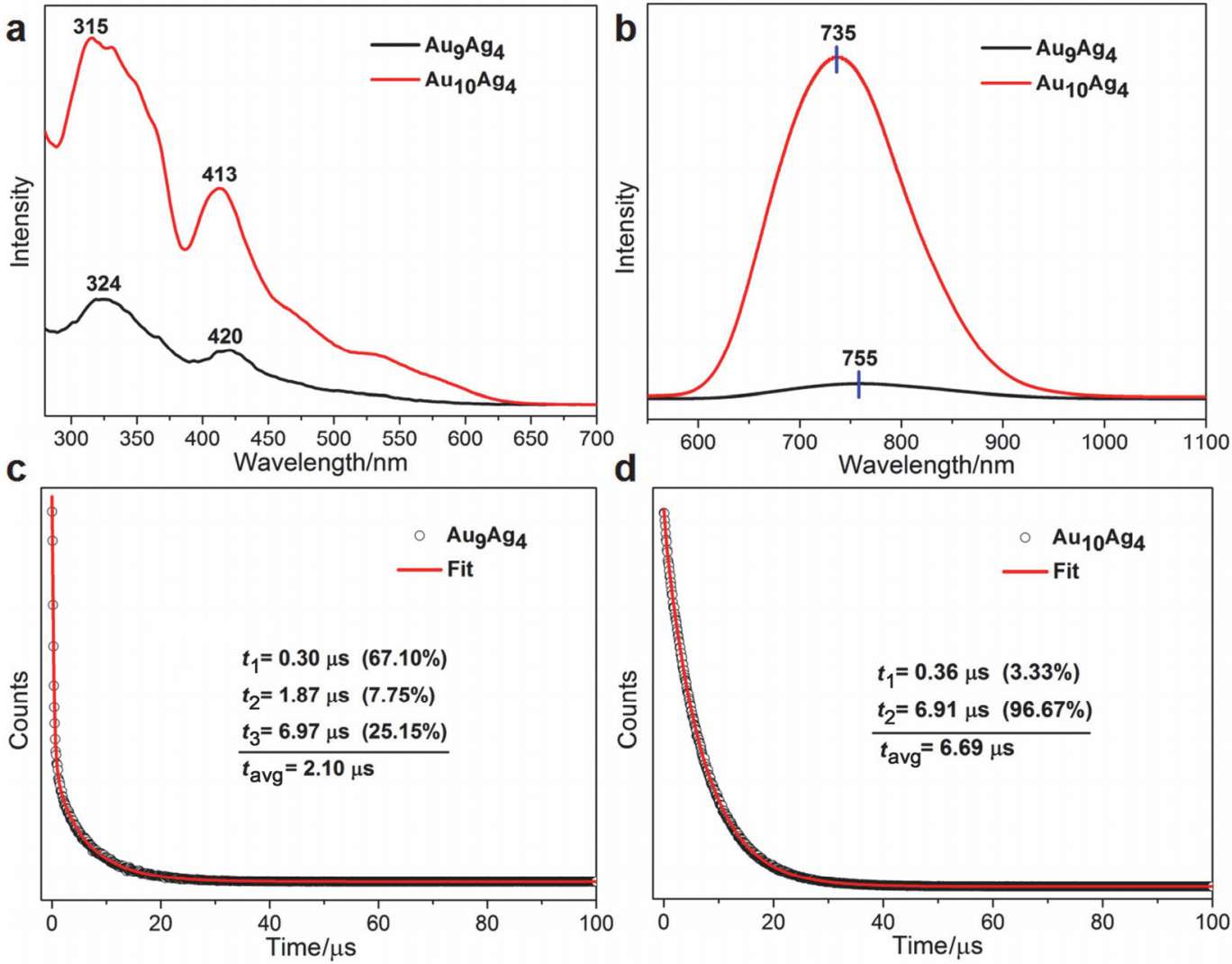

图 $4 \mathrm{Au}_{9} \mathrm{Ag}_{4} \mathrm{NC}$ 和 $\mathrm{Au}_{10} \mathrm{Ag}_{4} \mathrm{NC}$ 在乙腈中的 $\mathrm{PL}$ 激发光谱 $\left(\lambda_{\mathrm{EM}}=750 \mathrm{~nm}\right)(\mathrm{a})$ 和发射光谱 $\left(\lambda_{\mathrm{EX}}=405 \mathrm{~nm}\right)(\mathrm{b}) . \mathrm{Au}_{9} \mathrm{Ag}_{4} \mathrm{NC}(\mathrm{c})$ 和 $\mathrm{Au}_{10} \mathrm{Ag}_{4} \mathrm{NC}(\mathrm{d})$ 在乙腈 中的 PL 寿命衰减图

Figure 4 PL excitation ( $\lambda_{\mathrm{EM}}=750 \mathrm{~nm}$ ) (a) and emission spectra $\left(\lambda_{\mathrm{EX}}=405 \mathrm{~nm}\right)$ (b) of $\mathrm{Au}_{9} \mathrm{Ag}_{4} \mathrm{NC}$ and $\mathrm{Au}_{10} \mathrm{Ag}_{4} \mathrm{NC}$ in acetonitrile. PL lifetime decay diagrams of $\mathrm{Au}_{9} \mathrm{Ag}_{4} \mathrm{NC}$ (c) and $\mathrm{Au}_{10} \mathrm{Ag}_{4} \mathrm{NC}$ (d) in acetonitrile
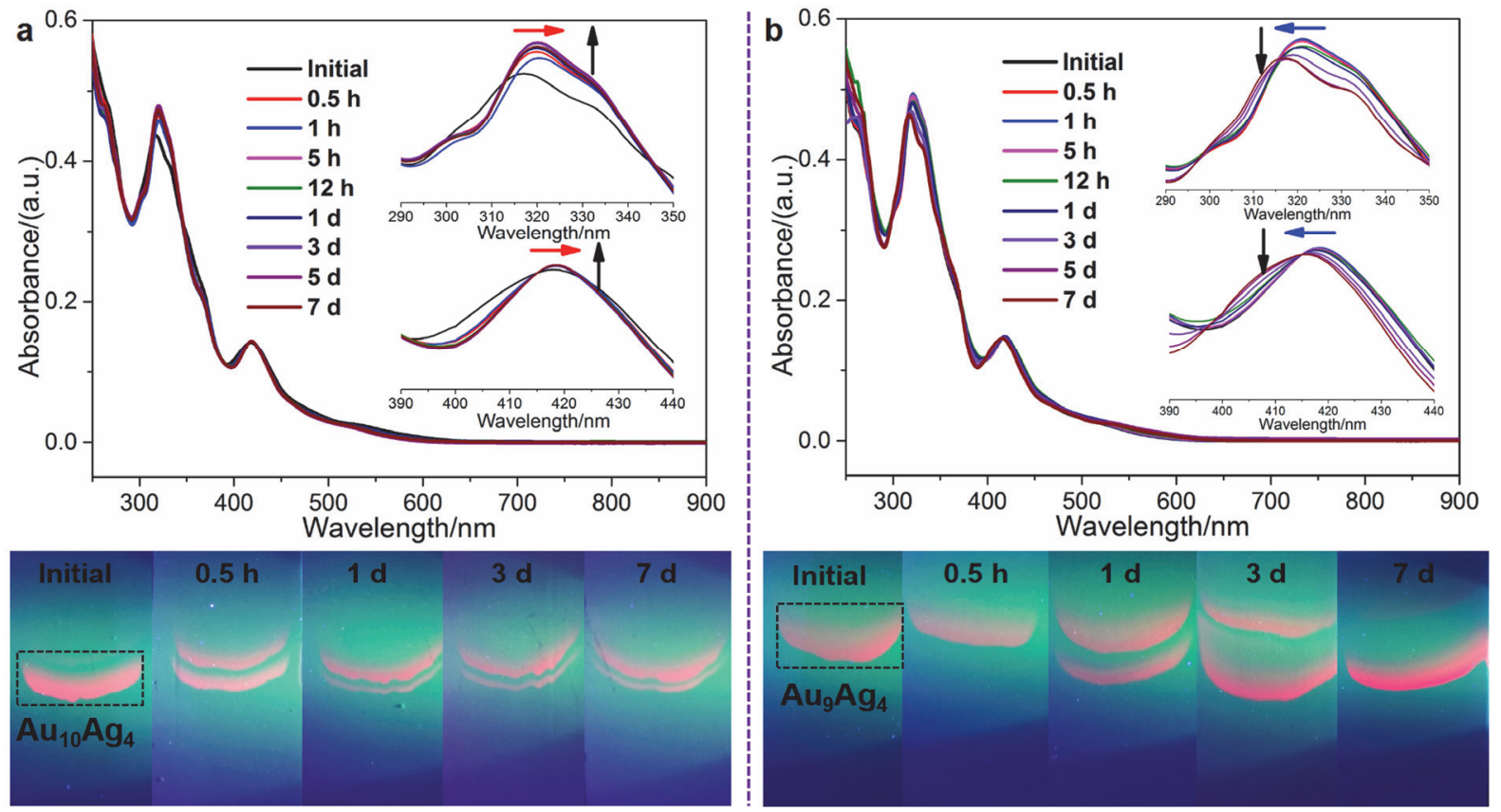

图 5 (a) 通过 UV/Vis/NIR 吸收光谱和制备型薄层色谱(PTLC)监测 $25{ }^{\circ} \mathrm{C}$ 在二氯甲烷中 $\mathrm{Au}_{10} \mathrm{Ag}_{4} \mathrm{NC}$ 向 $\mathrm{Au}_{9} \mathrm{Ag}_{4} \mathrm{NC}$ 的转变. (b) 通过 UV/Vis/NIR 吸收光谱和 PTLC 监测 $60{ }^{\circ} \mathrm{C}$ 下在乙醇中 $\mathrm{Au}_{9} \mathrm{Ag}_{4} \mathrm{NC}$ 向 $\mathrm{Au}_{10} \mathrm{Ag}_{4} \mathrm{NC}$ 的转变

Figure 5 (a) Conversion of $\mathrm{Au}_{10} \mathrm{Ag}_{4} \mathrm{NC}$ to $\mathrm{Au}_{9} \mathrm{Ag}_{4} \mathrm{NC}$ at $25{ }^{\circ} \mathrm{C}$ in dichloromethane monitored by UV/Vis/NIR absorption spectra and preparative thin layer chromatography (PTLC). (b) Conversion of $\mathrm{Au}_{9} \mathrm{Ag}_{4} \mathrm{NC}$ to $\mathrm{Au}_{10} \mathrm{Ag}_{4} \mathrm{NC}$ at $60{ }^{\circ} \mathrm{C}$ in ethanol monitored by UV/Vis/NIR absorption spectra and PTLC 
了它们的原子结构, 并首次在金属纳米团簇中发现了 RP-Au-PR 结构. 值得一提的是, 该结构可以自行生成 和消失, 取决于团簇周围的环境(溶剂等). 这种自生的 结构促使纳米团簇的 PL 增强了 19 倍, 可归因于电荷转 移和结构刚性的增强. 因此, 这项工作为金银纳米团簇 的结构和 PL 性质提供了新的视角, 对于理解金属纳米 才簇的构效关系和性能的改进具有重要的启示, 并有望 促进后续更多关于金属纳米团簇材料合成、结构、性能 和应用的研究.

\section{References}

[1] Jin, R.; Zeng, C.; Zhou, M.; Chen, Y. Chem. Rev. 2016, 116, 10346.

[2] Chakraborty, I.; Pradeep, T. Chem. Rev. 2017, 117, 8208.

[3] Wan, X.-K.; Yuan, S.-F.; Lin, Z.-W.; Wang, Q.-M. Angew. Chem. Int. Ed. 2014, 53, 2923.

[4] Si, W.-D.; Li, Y.-Z.; Zhang, S.-S.; Wang, S.; Feng, L.; Gao, Z.-Y.; Tung, C.-H.; Sun, D. ACS Nano 2021, 15, 16019.

[5] Antonello, S.; Perera, N. V.; Ruzzi, M.; Gascón, J. A.; Maran, F. J. Am. Chem. Soc. 2013, 135, 15585.

[6] Li, Q.; Zhou, M.; So, W. Y.; Huang, J.; Li, M.; Kaufffman, D. R.; Cotlet, M.; Higaki, T.; Peteanu, L. A.; Shao, Z.; Jin, R. J. Am. Chem. Soc. 2019, 141, 5314.

[7] Wu, Z.; Yao, Q.; Chai, O. J. H.; Ding, N.; Xu, W.; Zang, S.; Xie, J. Angew. Chem. Int. Ed. 2020, 59, 9934.

[8] Jin, F.; Dong, H.; Zhao, Y.; Zhuang, S.; Liao, L.; Yan, N.; Gu, W.; Zha, J.; Yuan, J.; Li, J.; Deng, H.; Gan, Z.; Yang, J.; Wu, Z. Acta Chim. Sinica 2020, 78, 407 (in Chinese). (金凤鸣, 董宏伟, 赵燕, 庄胜利，廖玲文，间楠，古万苗，查珺，袁金云，李进，邓海腾， 甘自保, 杨金龙, 伍志鲲, 化学学报, 2020, 78, 407.)

[9] Yamazoe, S.; Koyasu, K.; Tsukuda, T. Acc. Chem. Res. 2014, 47, 816.

[10] Liu, C.; Abroshan, H.; Yan, C.; Li, G.; Haruta, M. ACS Catal. 2016, 6,92 .

[11] Li, M.-B.; Tian, S.-K.; Wu, Z. Chin. J. Chem. 2017, 35, 567.

[12] Yan, J.; Teo, B. K.; Zheng, N. Acc. Chem. Res. 2018, 51, 3084.

[13] Zhuang, S.; Chen, D.; Liao, L.; Zhao, Y.; Xia, N.; Zhang, W.; Wang, C.; Yang, J.; Wu, Z. Angew. Chem. Int. Ed. 2020, 59, 3073.

[14] Yang, X.; Yang, M.; Pang, B.; Vara, M.; Xia, Y. Chem. Rev. 2015, $115,10410$.

[15] Chen, L.; Zhang, Y.; Jiang, H.; Wang, X.; Liu, C. Chin. J. Chem. 2016, 34, 589 .

[16] Gao, G.; Gong, D.; Zhang, M.; Sun, T. Acta Chim. Sinica 2016, 74, 363 (in Chinese). (高冠斌, 龚德君, 张明曦, 孙涛垒, 化学学报, 2016, 74, 363.)

[17] Lin, R.; Chen, Y.; Tao, G.; Pei, X.; Liu, F.; Li, N. Acta Chim. Sinica 2017, 75, 1103 (in Chinese). (林若韵, 陈阳, 陶广宇, 裴晓静, 刘
锋, 李娜, 化学学报, 2017, 75, 1103.)

[18] Jia, T.-T.; Yang, G.; Mo, S.-J.; Wang, Z.-Y.; Li, B.-J.; Ma, W.; Guo, Y.-X.; Chen, X.; Zhao, X.; Liu, J.-Q.; Zang, S.-Q. ACS Nano 2019, 13,8320 .

[19] Wen, F.; Dong, Y.; Feng, L.; Wang, S.; Zhang, S.; Zhang, X. Anal. Chem. 2011, 83, 1193.

[20] George, A.; Shibu, E. S.; Maliyekkal, S. M.; Bootharaju, M. S.; Pradeep, T. ACS Appl. Mater. Interfaces 2012, 4, 639.

[21] Yuan, X.; Luo, Z.; Yu, Y.; Yao, Q.; Xie, J. Chem. - Asian J. 2013, 8 , 858.

[22] Zhang, L.; Wang, E. Nano Today 2014, 9, 132.

[23] Qin, H.-N.; Wang, Z.-Y.; Zang, S.-Q. Acta Chim. Sinica 2021, 79 , 1037 (in Chinese). (秦浩男, 王朝阳, 歲双全, 化学学报, 2021, 79, 1037.)

[24] McPartlin, M.; Mason, R.; Malatesta, L. J. Chem. Soc. D 1969, 334.

[25] Briant, C. E.; Theobald, B. R. C.; White, J. W.; Bell, L. K.; Mingos, D. M. P.; Welch, A. J. J. Chem. Soc., Chem. Commun. 1981, 201

[26] Teo, B. K.; Hong, M. C.; Zhang, H.; Huang, D. B. Angew. Chem. Int. Ed. 1987, 26, 897.

[27] Kamei, Y.; Shichibu, Y.; Konishi, K. Angew. Chem. Int. Ed. 2011 $50,7442$.

[28] Chen, J.; Zhang, Q.-F.; Bonaccorso, T. A.; Williard, P. G.; Wang, L.-S. J. Am. Chem. Soc. 2014, 136, 92.

[29] Zhu, M.; Li, M.; Yao, C.; Xia, N.; Zhao, Y.; Yan, N.; Liao, L.; Wu, Z. Acta Phys.-Chim. Sin. 2018, 34, 792 (in Chinese). (祝敏, 李漫波, 姚传好, 夏楠, 赵燕, 间楠, 廖玲文, 伍志鲲, 物理化学学报, 2018, 34, 792.)

[30] Jadzinsky, P. D.; Calero, G.; Ackerson, C. J.; Bushnell, D. A.; Kornberg, R. D. Science 2007, 318, 430.

[31] Zhu, M.; Aikens, C. M.; Hollander, F. J.; Schatz, G. C.; Jin, R. J. Am Chem. Soc. 2008, 130, 5883.

[32] Wu, Z.; Jin, R. Nano Lett. 2010, 10, 2568.

[33] Pyo, K.; Thanthirige, V. D.; Kwak, K.; Pandurangan, P.; Ramakrishna, G.; Lee, D. J. Am. Chem. Soc. 2015, 137, 8244.

[34] Gan, Z.; Lin, Y.; Luo, L.; Han, G.; Liu, W.; Liu, Z.; Yao, C.; Weng, L.; Liao, L.; Chen, J.; Liu, X.; Luo, Y.; Wang, C.; Wei, S.; Wu, Z. Angew. Chem. Int. Ed. 2016, 55, 11567.

[35] Wu, Z.; Suhana, J.; Jin, R. J. Mater. Chem. 2009, 19, 622.

[36] Xia, N.; Wu, Z. Chem. Sci. 2021, 12, 2368.

[37] Shichibu, Y.; Konishi, K. Small 2010, 6, 1216.

[38] Walter, M.; Akola, J.; Lopez-Acevedo, O.; Jadzinsky, P. D.; Calero, G.; Ackerson, C. J.; Whetten, R. L.; Grönbeck, H.; Häkkinen, H. Proc. Natl. Acad. Sci. 2008, 105, 9157.

[39] Wu, Z.; Jin, R. Chem. Eur. J. 2011, 17, 13966.

[40] Dhayal, R. S.; Liao, J.-H.; Liu, Y.-C.; Chiang, M.-H.; Kahlal, S.; Saillard, J.-Y.; Liu, C.-W. Angew. Chem. Int. Ed. 2015, 54, 3702.

[41] Jin, S.; Zou, X.; Xiong, L.; Du, W.; Wang, S.; Pei, Y.; Zhu, M. Angew. Chem. Int. Ed. 2018, 57, 16768.

[42] Weerawardene, K. D. M.; Pandeya, P.; Zhou, M.; Chen, Y.; Jin, R.; Aikens, C. M. J. Am. Chem. Soc. 2019, 141, 18715.

(Yang, X.) 